\title{
FREE BOUNDARY FLUID SYSTEMS IN A SEMIGROUP APPROACH AND OSCILLATORY BEHAVIOR*
}

\author{
BEN SCHWEIZER ${ }^{\dagger}$
}

\begin{abstract}
We consider the free boundary problem of a liquid drop with viscosity and surface tension. We study the linearized equations with semigroup methods to get existence results for the nonlinear problem. The spectrum of the generator is computed. Large surface tension creates nonreal eigenvalues, and an exterior force results in a Hopf bifurcation. The methods are used to study wind-generated surface waves.
\end{abstract}

Key words. Hopf bifurcation, viscous fluid, free boundary

AMS subject classifications. 35Q30, 76D33

\section{PII. S0036141096299892}

1. Introduction. We consider two examples of a finite mass of viscous fluid with a free boundary. In contrast to the case of a fixed domain, the fluid is capable of showing damped oscillations. Due to surface tension the surface area carries potential energy, and oscillations correspond to an exchange of energy between its kinetic and its potential form. The time-dependent problem has parabolic and hyperbolic features, and Beale calls it "mixed in character." The work at hand contributes to the study of this dynamical system.

We assume the system to be close to a stationary solution. The theory is written down for an almost spherical liquid drop but applies also to water in a container with periodic lateral boundaries. We rewrite the equations in semigroup form; the study of the generator reveals some nonstandard properties regarding the choice of function spaces, the spectrum, and the resolvent.

In sections 2-4 we derive an existence theory using the language of semigroup theory and maximal regularity results. In sections 5-7 we study qualitative properties of the spectrum of the generator, such as nonreal or (with an external forcing) imaginary eigenvalues. The two parts interact: the existence theory allows the proof of a Hopf bifurcation in two examples.

The existence theory begins with a proof that the spectrum of the generator consists of eigenvalues and is contained in a sector of the complex plane. Therefore, a natural idea is to apply the semigroup theory for sectorial operators. But the analysis of the resolvent shows that the problem does not fit into this framework: the estimates are valid only on a subspace. On the other hand, due to the kinematic boundary condition, the nonlinearity is always contained in the same subspace. We show that the methods of semigroup theory can be adapted and derive an existence result for the linear problem in section 3 and for the nonlinear problem in section 4. The solutions provide differentiable flows on a Banach manifold. This will be the setting to prove a Hopf bifurcation in section 6 .

* Received by the editors February 23, 1996; accepted for publication (in revised form) May 2, 1996.

http://www.siam.org/journals/sima/28-5/29989.html

$\dagger$ Institut für Angewandte Mathematik, Universität Heidelberg, INF 294, 69120 Heidelberg, Germany (ben.schweizer@iwr.uni-heidelberg.de). This research was done in part while the author visited the Courant Institute for Mathematical Sciences, New York, supported by the Deutsche Forschungsgemeinschaft. 
Regarding other works, we wish to mention first Beale [2], who studied infinite domains. He derives the resolvent estimate with the help of Fourier transforms. His proof is considerably longer than ours, since the infinite domain corresponds to a continuous spectrum.

Concerning finite domains, we refer to the numerous works of Solonnikov, who gave the first existence result. He derives estimates with methods from potential theory, after having transformed the equations in a half-space [15]. We mention a related article on Hopf bifurcation in a two-phase fluid system by Renardy and Joseph [13]. In their more physical model the transversal crossing of eigenvalues appears as an assumption. They do not treat the initial value problem. For a more geometric approach, see Bemelmans [4] and Wagner [17].

In the second part of this article we prove qualitative properties of the spectrum. We describe the basic idea for the case where we have only one physical parameter, a nondimensional surface tension $\beta$. We are interested in how an eigenvalue $\lambda$ of the operator $\mathcal{L}_{\beta}$ depends on $\beta$. We do this indirectly. For some function $\tilde{\beta}$ every complex number $\lambda$ is an eigenvalue of the operator $\mathcal{L}_{\tilde{\beta}(\lambda)}$. The study of the function $\tilde{\beta}$ on the real axis gives us insight into the behavior of $\lambda(\beta)$.

In section 5 , we see that for vanishing surface tension the spectrum of $\mathcal{L}$ consists of both the Stokes eigenvalues in a fixed domain and the interfacial eigenvalue 0. For a fixed interfacial eigenmode with increasing $\beta$, the first two eigenvalues move towards each other and must leave the real axis, while the other eigenvalues remain trapped in fixed intervals.

We use the same general idea in two examples with an exterior force. In section 6 , we assume that the liquid drop experiences negative damping. We can count the eigenvalues inside a ball and prove that if the force reaches a critical strength a pair of eigenvalues crosses transversally the imaginary axis. Using the existence results, we can prove a Hopf bifurcation.

In section 7 we apply the idea to a model for the generation of water waves by wind. A strong wind leads to a Hopf bifurcation. We also gain insight into the shape of the eigenfunctions for strong wind: two of them show the structure of an ideal fluid; the other, approximate Stokes eigenfunctions. There are works (e.g., [10], [11]) that give asymptotic formulas for the eigenvalues for a vanishing exterior force. Our results confirm their qualitative properties and provide additional mathematical insight and proofs.

2. The liquid-drop equations. We first collect the nonlinear equations describing a liquid drop. Let $\Omega$ be the subdomain of $\mathbb{R}^{3}$ occupied by liquid. The velocity field and the pressure within the liquid drop are denoted by $u$ and $p$, respectively. The exterior normal vector of $\partial \Omega$ is denoted by $n^{\Omega}$; tangential vectors are denoted by $\tau_{i}^{\Omega}$. We use the dimensionless viscosity $\nu$.

The surface tension will become important. The physical quantity is a number $\beta>0$ : if the surface has a mean curvature $H(\eta)$, then the surface tension creates a pressure $2 \beta H(\eta)$.

We introduce the strain-tensor

$$
\left(S_{u}\right)_{i j}=\frac{1}{2}\left(\partial_{i} u_{j}+\partial_{j} u_{i}\right)
$$

and the product $\left(S_{u}\right):\left(S_{w}\right):=\sum_{i, j}\left(S_{u}\right)_{i j}\left(S_{w}\right)_{i j}$, and we define additionally

$$
S_{u}^{n}:=n \cdot S_{u} \cdot n
$$


We deal with a free boundary problem. The unknown functions are not only $u$ and $p$ but also the domain $\Omega$. We assume small perturbations of the unit sphere and parametrize the surface of the liquid drop with a function $\eta: S^{2} \rightarrow \mathbb{R}$. The domain occupied by liquid is

$$
\Omega(t)=\left\{r \xi \in \mathbb{R}^{3} \mid \xi \in S^{2}, 0 \leq r<1+\eta(\xi)\right\} .
$$

The velocity field is a function

$$
u(t, .): \Omega(t) \rightarrow \mathbb{R}^{3} .
$$

In the interior, the following Navier-Stokes equations hold:

$$
\begin{aligned}
\partial_{t} u+(u \cdot \nabla) u-\nu \Delta u+\nabla p & =0, \\
\nabla \cdot u & =0 .
\end{aligned}
$$

The boundary conditions are the geometric condition that $\eta$ always parametrizes the surface, the additional pressure created by surface tension, the condition of vanishing tangential stress, plus initial conditions:

$$
\begin{aligned}
\partial_{t} \eta+\left(\partial_{\varphi} \eta\right) u_{\varphi}+\left(\partial_{\vartheta} \eta\right) u_{\vartheta} & =u_{r}, \\
p-2 \nu n^{\Omega} \cdot S_{u} \cdot n^{\Omega} & =2 \beta H(\eta), \\
\tau_{i}^{\Omega} \cdot S_{u} \cdot n^{\Omega} & =0 \\
(u, \eta)(t=0) & =\left(u_{0}, \eta_{0}\right) .
\end{aligned}
$$

Equation (3) can be derived by considering a particle at the boundary with position $(r(t), \varphi(t), \vartheta(t))$ in spherical coordinates using $\eta(t, \varphi(t), \vartheta(t))=r(t)$.

We will return to these nonlinear equations in section 4 . We now give the linearization of the problem in $u=0, \eta=0$. We replace the domain $\Omega$ by the unit ball $B^{3} \cdot n^{\Omega}$ and $\tau_{i}^{\Omega}$ are replaced by the normal and tangential vectors of the unit sphere, $n(\xi)=\xi$ and $\tau_{i}$. The radial velocity will now be written as $\left.u_{n}\right|_{\partial}=\left.n \cdot u\right|_{\partial}$.

The linearization of the mean curvature of $\partial \Omega=\left\{\xi(1+\eta(\xi)) \mid \xi \in S^{2}\right\}$ is denoted by $-{ }_{2}^{1} \Delta \eta$. With the Laplace-Beltrami operator of the sphere $\Delta_{B}$, there holds

$$
\Delta=\Delta_{B}+2 \cdot i d
$$

The linearized equations are

$$
\begin{aligned}
u: B^{3} \rightarrow \mathbb{R}^{3}, \quad p: B^{3} \rightarrow \mathbb{R}, & \quad \eta: S^{2} \rightarrow \mathbb{R} \\
\frac{d}{d t} u-\nu \Delta u+\nabla p & =0 \\
\nabla \cdot u & =0 \\
d & =\left.u_{n}\right|_{\partial} \\
d t & =\beta \Delta \eta \\
\left.\left(-p+2 \nu S_{u}^{n}\right)\right|_{\partial} & =\beta \Delta) \\
\left.\left(\tau_{i} \cdot S_{u} \cdot n\right)\right|_{\partial} & =0 .
\end{aligned}
$$

In the equation for the pressure, we omitted the constant pressure induced by the surface tension of the unit sphere.

Before we start the analysis of the linearized liquid-drop equations, we collect some facts concerning the Stokes equation. We write $H^{r}=H^{r, 2}$ for Sobolev spaces. 
If the domain is the unit ball $B=B^{3}$, we often omit this argument: $H^{r}=H^{r}\left(B^{3}\right)$. The Stokes operator $A:(u, p) \mapsto(-\nu \Delta u+\nabla p, \nabla \cdot u)$ is elliptic in the sense of Agmon, Douglis, and Nirenberg [1] with any of the boundary conditions

$$
\begin{array}{ll} 
& \left.u\right|_{\partial}=0, \\
\text { or } \quad & \left.u_{n}\right|_{\partial}=0,\left.\quad \tau \cdot S_{u}\right|_{\partial} \cdot n=0, \\
\text { or }\left.\quad\left(p-2 \nu S_{u}^{n}\right)\right|_{\partial}=0,\left.\quad \tau \cdot S_{u}\right|_{\partial} \cdot n=0 .
\end{array}
$$

Solutions to inhomogeneous boundary data have maximal regularity.

We introduce the operator

$$
\mathcal{H}: H^{r-1 / 2}\left(S^{2}\right) \rightarrow H^{r}\left(B^{3}\right),
$$

which maps a function to its harmonic extension.

We will use integration by parts in the following form.

LEMma 2.1. For smooth functions $u, w: B^{3} \rightarrow \mathbb{R}^{3}$ with $\nabla \cdot u=\nabla \cdot w=0$ and $\tau \cdot S_{u} \cdot n=0$,

$$
2 \int_{B} S_{u}: S_{w}=\int_{B}\left\{-\Delta u+\nabla \mathcal{H}\left(2 S_{u}^{n}\right)\right\} \cdot w
$$

We now return to the liquid-drop equations. Our aim is to write the linear liquiddrop equations in the form $\frac{d}{d t} x+\mathcal{L} x=0$ and to satisfy the boundary conditions by the choice of appropriate function spaces.

We start by rewriting the boundary condition for the pressure. The pressure $p$ is a harmonic function; therefore

$$
p=\mathcal{H}\left(\left.2 \nu S_{u}^{n}\right|_{\partial}\right)-\mathcal{H}(\beta \Delta \eta) .
$$

The physical quantities volume, momentum, and angular momentum are conserved. We use this fact in the definition of the function spaces.

The following point of view is useful: the first eigenspace of $\Delta_{B}$ corresponds to constant functions $\Phi(x)=a$; the second eigenspace, to translations $\Phi(x)=b \cdot x$. The physical conditions imply that the projection of $\eta$ onto the first two eigenspaces of $\Delta_{B}$ vanishes.

Definition 2.2. Define the Hilbert spaces

$$
\begin{aligned}
Y^{r} & :=\left\{u \in H^{r}\left(B^{3}\right)^{3} \mid \nabla \cdot u=0 ; \int_{B^{3}} u=0 ; \int_{B^{3}} u \wedge \gamma=0 \forall \gamma \in \mathbb{R}^{3}\right\}, \\
X^{r} & :=\left\{(u, \eta) \in Y^{r} \times H^{r+1-1 / 2}\left(S^{2}\right) \mid \int_{S^{2}} \eta=0 ; \int_{S^{2}} n \cdot \eta=0\right\}, \\
\tilde{X}^{r} & :=\left\{(u, \eta) \in X^{r}\left|n \cdot S_{u}(z) \cdot \tau\right|_{\partial}=0 \forall \tau \in T_{z} S^{2}\right\}
\end{aligned}
$$

and the operator

$$
\mathcal{L}: X^{r} \rightarrow X^{r}, \quad \tilde{X}^{r} \supset \mathcal{D}(\mathcal{L}) \supset \tilde{X}^{r+2}
$$

by

$$
\mathcal{L}\left(\begin{array}{c}
u \\
\eta
\end{array}\right):=\left(\begin{array}{c}
-\nu \Delta u+\nabla \mathcal{H}\left(\left.2 \nu S_{u}^{n}\right|_{\partial}\right)-\nabla \mathcal{H}(\beta \Delta \eta) \\
-\left.u_{n}\right|_{\partial}
\end{array}\right)
$$


Easy calculations show that $\mathcal{L}$ maps to $X$ : the liquid drop does not start to move its center of mass, it does not start to rotate, and it keeps its volume.

The linearized liquid-drop equation reads

$$
\frac{d}{d t} x+\mathcal{L} x=0, \quad x \in \tilde{X}
$$

In the case of a pure rotation the integral $\int_{B}\left|S_{u}\right|^{2}$ vanishes without $u$ being a constant. But in our function spaces a Korn inequality holds: there exists a constant $C_{K}$ such that for all $u \in Y$

$$
\frac{1}{C_{K}}\|D u\|_{L^{2}}^{2} \leq 2 \nu \int_{B^{3}}\left|S_{u}\right|^{2} \leq C_{K}\|D u\|_{L^{2}}^{2}
$$

See, e.g., [16]. Here $\|D u\|_{L^{2}}$ may be replaced by $\|u\|_{H^{1}}$ because the mean of $u$ vanishes.

Definition 2.3 (energy norms). For functions $u, v: B^{3} \rightarrow \mathbb{R}^{3}$ and $\eta, \sigma: S^{2} \rightarrow \mathbb{R}$ we define

$$
\begin{aligned}
\langle u, v\rangle_{E} & :=\int_{B} \bar{u} \cdot v \\
\langle\eta, \sigma\rangle_{E} & :=\beta \int_{S}(-\Delta \bar{\eta}) \cdot \sigma \\
\left\langle\left(\begin{array}{c}
u \\
\eta
\end{array}\right),\left(\begin{array}{c}
v \\
\sigma
\end{array}\right)\right\rangle_{E} & :=\langle u, v\rangle_{E}+\langle\eta, \sigma\rangle_{E} .
\end{aligned}
$$

The corresponding norms are denoted by $\|.\|_{E}$.

LEMma 2.4 (position of eigenvalues of $\mathcal{L}$ ). Let $(u, \eta) \in \tilde{X}^{2}$ be an eigenvector of $\mathcal{L}$ with eigenvalue $\mu$. Then

$$
\begin{aligned}
& \operatorname{Re}(\mu)\|(u, \eta)\|_{E}^{2}=2 \nu \int_{B}\left|S_{u}\right|^{2}, \\
& \operatorname{Im}(\mu)\|(u, \eta)\|_{E}^{2}=2 \beta \operatorname{Im}\left(\left.\int_{S} u_{n}\right|_{\partial} \Delta \bar{\eta}\right) .
\end{aligned}
$$

In the case of $\operatorname{Im}(\mu) \neq 0$ the following energy equality holds:

$$
\|u\|_{E}^{2}=\|\eta\|_{E}^{2}=\frac{1}{2}\|(u, \eta)\|_{E}^{2} .
$$

Proof.

$$
\begin{aligned}
\mu\left\|\left(\begin{array}{c}
u \\
\eta
\end{array}\right)\right\|_{E}^{2}= & \left\langle\left(\begin{array}{c}
u \\
\eta
\end{array}\right), \mathcal{L}\left(\begin{array}{c}
u \\
\eta
\end{array}\right)\right\rangle_{E} \\
= & \left\langle\left(\begin{array}{c}
u \\
\eta
\end{array}\right),\left(\begin{array}{c}
-\nu \Delta u+\nabla \mathcal{H}\left(2 \nu S_{u}^{n}\right)-\nabla \mathcal{H}(\beta \Delta \eta) \\
-\left.u_{n}\right|_{\partial}
\end{array}\right)\right\rangle_{E} \\
= & \int_{B}\left\{\bar{u} \cdot\left(-\nu \Delta u+\nabla \mathcal{H}\left(2 \nu S_{u}^{n}\right)\right)\right\} \\
& -\int_{B} \bar{u} \nabla \mathcal{H}(\beta \Delta \eta)-\left.\beta \int_{S}(-\Delta \bar{\eta})\left(u_{n}\right)\right|_{\partial} \\
= & 2 \nu \int_{B}\left|S_{u}\right|^{2}+\beta \int_{S}\left\{\left.u_{n}\right|_{\partial} \Delta \bar{\eta}-\left.\bar{u}_{n}\right|_{\partial} \Delta \eta\right\} .
\end{aligned}
$$


This implies the assertion on the real and the imaginary part of $\mu$.

To prove the energy equality we use the second part of the eigenvalue equation, $-\left.u_{n}\right|_{\partial}=\mu \eta$ :

$$
\begin{aligned}
\operatorname{Im}(\mu)\left\|\left(\begin{array}{c}
u \\
\eta
\end{array}\right)\right\|_{E}^{2} & =2 \beta \operatorname{Im}\left(\left.\int_{S} u_{n}\right|_{\partial} \Delta \bar{\eta}\right) \\
& =2 \operatorname{Im}(\mu)\|\eta\|_{E}^{2} .
\end{aligned}
$$

Using the properties of the Stokes operator one easily proves the following lemma for $\beta \neq 0$.

LEMma 2.5. The operator $\mathcal{L}^{-1}: X^{r} \rightarrow \tilde{X}^{r+1}$ is bounded.

We point out that $\mathcal{L}^{-1}: X^{0} \rightarrow \tilde{X}^{2}$ is not bounded: let $(u, \eta)$ solve $\mathcal{L}(u, \eta)=$ $(0, g)$. A bound for $\|u\|_{H^{2}}$ would imply $g=\left.u_{n}\right|_{\partial} \in H^{3 / 2,2}\left(S^{2}\right)$. But a priori, only $g \in H^{1 / 2,2}\left(S^{2}\right)$ holds.

The Lumer-Phillips theorem implies the following lemma.

Lemma 2.6. $\mathcal{L}$ generates a $C^{0}$-semigroup in the space $X_{E}$ corresponding to the energy norms.

We want to split $X$ into a direct sum of $\mathcal{L}$-invariant subspaces $\left(X_{n}\right)_{n \in \mathbb{N}}$ according to spherical harmonics. The functions $\left\{\psi_{n, k} \mid n \in \mathbb{N}, k \in\{-n, \ldots, n\}\right\}$ shall span $\Psi_{n}$, the $n$th eigenspace of the Laplace-Beltrami operator of $S^{2}$. We denote the corresponding eigenvalue by $\Lambda_{n}>0$ and the eigenvalue of $-\Delta$ by $\Lambda_{n}=\Lambda_{n}-2$. Let $\nu$ be the normal vector, $\nabla_{T}$ the tangential gradient, and $\nabla_{T}^{\perp}=\nu \wedge \nabla$ the orthogonal tangential gradient.

A vector field is in $X_{n}$ if on any sphere of radius $r$ the function can be represented by $\psi_{n, k} \nu, \nabla_{T} \psi_{n, k}$, and $\nabla_{T}^{\frac{1}{T}} \psi_{n, k}, k \in\{-n, \ldots, n\}$.

Proposition 2.7. The spectrum of $\mathcal{L}$ consists only of eigenvalues which are contained in a sector

$$
S_{C}:=\{\mu|\quad| \operatorname{Im}(\mu) \mid<C \operatorname{Re}(\mu)\} .
$$

Proof. By Lemma $2.5, \mathcal{L}$ has a compact resolvent and therefore a pure point spectrum. We prove that eigenvalues are contained in a sector $S_{C}$. Let $\mu$ be an eigenvalue with eigenvector $(u, \eta) \in \tilde{X}_{k}$. If $\operatorname{Im}(\mu)=0$, then $\mu$ is contained in any sector $S_{C}$. We can therefore assume $\operatorname{Im}(\mu) \neq 0$.

We use Lemma 2.4 and the fact that $u$ depends on the radius $r$ like $e^{\sqrt{ } \Lambda_{k}+|\mu| r}$.

$$
\begin{aligned}
|\operatorname{Im}(\mu)|\left\|\left(\begin{array}{c}
u \\
\eta
\end{array}\right)\right\|_{E}^{2} & =2 \beta\left|\operatorname{Im}\left(\left.\int_{S} u_{n}\right|_{\partial} \Delta \bar{\eta}\right)\right| \\
& \leq \beta \int_{S}|\Delta \eta|^{2}+\left.\beta \int_{S}\left|u_{n}\right|_{\partial}\right|^{2} \\
& \leq\left|\Lambda_{k}\right|\|\eta\|_{E}^{2}+\beta C_{T} \sqrt{\Lambda_{k}+|\mu|\|u\|_{L^{2}(B)}^{2}} .
\end{aligned}
$$

Therefore,

$$
|\operatorname{Im}(\mu)| \leq C_{1}\left|\Lambda_{k}\right|+\beta C_{2} \operatorname{Re}(\mu) .
$$

Using Lemma 2.4 and the Korn inequality yields

$$
\operatorname{Re}(\mu)\left\|\left(\begin{array}{c}
u \\
\eta
\end{array}\right)\right\|_{E}^{2}=2 \nu \int_{B}\left|S_{u}\right|^{2}
$$




$$
\begin{aligned}
& \geq \frac{1}{C_{K}}\left\|\nabla_{T} u\right\|_{L^{2}}^{2} \\
& \geq \frac{1}{2 C_{K}}\left|\Lambda_{k}\right|\left\|\left(\begin{array}{c}
u \\
\eta
\end{array}\right)\right\|_{E}^{2} .
\end{aligned}
$$

The assertion follows with the constant $C=2 C_{1} C_{K}+\beta C_{2}$.

3. An estimate for resolvents and the time-dependent problem. In this section we collect estimates for the resolvent. A first type of estimate concerns solutions $(u, \eta)$ of $(\lambda-\mathcal{L})(u, \eta)=(f, 0)$. Such estimates are known in similar contexts [2], [13]. We indicate how they can be derived more easily in our case of only one fluid in a compact domain.

As a corollary, we get a second type of estimate concerning the resolvent on the full space: we show that $\mathcal{L}$ is a sectorial operator.

To solve nonlinear equations, it will be necessary to increase the regularity of the function spaces. In this section we use $X=X^{r}$ and $\tilde{X}^{++}=\tilde{X}^{2+r}$ with $r=0$ or $r=2$.

TheOREM 3.1. There exists $C_{R}>0$ such that solutions $(u, \eta) \in \tilde{X}^{++}$of

$$
(\lambda-\mathcal{L})\left(\begin{array}{c}
u \\
\eta
\end{array}\right)=\left(\begin{array}{l}
f \\
0
\end{array}\right)
$$

with $\lambda \in \mathbb{C} \backslash S_{C}$ satisfy the regularity

$$
\|(u, \eta)\|_{X^{++}} \leq C_{R}\|(f, 0)\|_{X}
$$

and the resolvents estimate

$$
\|(u, \eta)\|_{X} \leq C_{R} \frac{1}{|\lambda|}\|(f, 0)\|_{X}
$$

Proof. We indicate the ideas of the proof. One writes the equation as

$$
\lambda u+\nu \Delta u-\nabla \mathcal{H}\left(2 \nu S_{u}^{n}\right)-{ }_{\lambda}^{1} \nabla \mathcal{H}\left(\left.\beta \Delta u_{n}\right|_{\partial}\right)=f .
$$

Testing with $\Delta_{B} \bar{u}$ and taking real and imaginary parts shows that $\left.\beta_{|\lambda|}^{1} \int_{S^{2}}\left|\Delta u_{n}\right|_{\partial}\right|^{2}$ and $\|u\|_{H^{2}}^{2}$ can be estimated by $\|f\|_{L^{2}}$ and $|\lambda|^{2}\|u\|_{L^{2}}^{2}$.

Testing (12) with $\bar{u}$ and taking the imaginary part yields

$$
\begin{aligned}
|\lambda|^{2}\|u\|_{L^{2}}^{2} & \leq \beta \int_{S^{2}} \bar{u}_{n} \Delta u_{n}+\text { const }\|f\|_{L^{2}}|\lambda|\|u\|_{L^{2}} \\
& \leq \beta C_{c}\|u\|_{L^{2}}^{1 / 2}\|u\|_{H^{2}}^{3 / 2}+\text { const }\|f\|_{L^{2}}^{2}+\frac{1}{2}|\lambda|^{2}\|u\|_{L^{2}}^{2} .
\end{aligned}
$$

This yields the estimates for $u$. Equation (12) provides the estimates for $\eta$.

Corollary 3.2. $\mathcal{L}$ is a sectorial operator on $X^{r+2}, r \geq 0$. The spectrum $\sigma(\mathcal{L})$ is contained in a sector $S_{C}$, and with a constant $M>0$, for every $\lambda \in \mathbb{C} \backslash S_{C}$,

$$
(\lambda-\mathcal{L})\left(\begin{array}{c}
u \\
\eta
\end{array}\right)=\left(\begin{array}{c}
f \\
g
\end{array}\right) \Rightarrow\left\|\left(\begin{array}{c}
u \\
\eta
\end{array}\right)\right\|_{X^{r+2}} \leq \frac{M}{|\lambda|}\left\|\left(\begin{array}{c}
f \\
g
\end{array}\right)\right\|_{X^{r+2}} .
$$


Proof. Instead of (12),

$$
\lambda u+\nu \Delta u-\nabla \mathcal{H}\left(2 \nu S_{u}^{n}\right)-\frac{1}{\lambda} \nabla \mathcal{H}\left(\left.\beta \Delta u_{n}\right|_{\partial}\right)=f-\frac{1}{\lambda} \nabla \mathcal{H}\left(\left.\beta \Delta g\right|_{\partial}\right) .
$$

For $f=0$ Theorem 3.1 yields

$$
\begin{aligned}
\|(u, \eta)\|_{X^{r+2}}+|\lambda|\|(u, \eta)\|_{X^{r}} & \leq \operatorname{const} \frac{1}{|\lambda|}\|g\|_{H^{r+3-1 / 2}\left(S^{2}\right)} \\
& \leq \operatorname{const} \frac{1}{|\lambda|}\|(0, g)\|_{X^{r+2}}
\end{aligned}
$$

The preceding corollary verifies one of the assumptions in the Hopf bifurcation theorem of [5]; nevertheless, that theorem cannot be applied since it assumes the nonlinearity to be of lower order.

Using Theorem 3.1 we can solve the initial value problem with the ideas of semigroup theory. Unlike in other approaches, this will provide a time- $t$ map for the nonlinear evolution system. In particular, this tool allows an elementary proof of a Hopf bifurcation theorem.

Definition 3.3. Let $I:=[0, T]$ be a fixed time interval, $T>0$. We introduce the spaces

$$
\begin{gathered}
Z:=C^{\alpha}(I, X), \\
\tilde{Z}^{++}:=C^{1, \alpha}(I, X) \cap C^{\alpha}\left(I, \tilde{X}^{++}\right) .
\end{gathered}
$$

For the regularity of the initial values we define

$$
D_{\mathcal{L}, \alpha}:=\left\{x \in X \mid \quad\|x\|_{K, \alpha}:=\left\|t \mapsto e^{-t \mathcal{L}} x\right\|_{Z^{++}}<\infty\right\} .
$$

As in the semigroup theory, we choose a path of integration $\Gamma$ in the complex plane containing $S_{C}$ and write functions in $\mathcal{L}$ as integrals over $\Gamma$. Following the standard lines we get the special semigroup estimates

$$
\begin{array}{r}
\left\|e^{-t \mathcal{L}}(f, 0)\right\|_{X}+\left\|t \mathcal{L} e^{-t \mathcal{L}}(f, 0)\right\|_{X}+\left\|(t \mathcal{L})^{2} e^{-t \mathcal{L}}(f, 0)\right\|_{X} \leq C\|(f, 0)\|_{X}, \\
\left\|\mathcal{L}^{-1} e^{-t \mathcal{L}}(f, 0)\right\|_{X^{++}}+\left\|t e^{-t \mathcal{L}}(f, 0)\right\|_{X^{++}}+\left\|t^{2} \mathcal{L} e^{-t \mathcal{L}}(f, 0)\right\|_{X^{++}}
\end{array}
$$

We can now prove a result of maximal regularity, i.e., solutions of $\partial_{t} x+\mathcal{L} x=F$ are in $Z^{++}$if $F$ is in $Z$. The underlying idea is taken from [6], which proves a regularity result in the case when the resolvent of the generator has optimal regularity properties.

Theorem 3.4. Let $F \in Z=C^{\alpha}(I, X)$ be of the form $F=\left(\begin{array}{l}f \\ 0\end{array}\right)$ and let $x_{0} \in \tilde{X}^{++}$satisfy the compatibility condition

$$
x_{0}-\mathcal{L}^{-1} F(0) \in D_{\mathcal{L}, \alpha} .
$$

Then the equation

$$
\begin{aligned}
\partial_{t} x+\mathcal{L} x & =\left(\begin{array}{l}
f \\
0
\end{array}\right), \\
x(0) & =x_{0}
\end{aligned}
$$


has a unique solution $x \in \tilde{Z}^{++}$bounded by

$$
\|x\|_{Z^{++}} \leq C_{1}(T, M, \alpha)\left\{\left\|x_{0}\right\|_{X^{++}}+\left\|x_{0}-\mathcal{L}^{-1} F(0)\right\|_{K, \alpha}+\|F\|_{Z}\right\} .
$$

The compatibility condition is always satisfied:

$$
\left\|x(t)-\mathcal{L}^{-1} F(t)\right\|_{K, \alpha} \leq C_{2}(T, M, \alpha) \cdot\left\{\left\|x_{0}\right\|_{X^{++}}+\left\|x_{0}-\mathcal{L}^{-1} F(0)\right\|_{K, \alpha}+\|F\|_{Z}\right\} .
$$

Proof. One proves that $x($.$) is in C^{\alpha}\left(I, X^{++}\right)$by decomposing $x(t)$ as

$$
\begin{aligned}
x(t)= & e^{-t \mathcal{L}} x_{0}+\int_{0}^{t} e^{-(t-s) \mathcal{L}} F(s) d s \\
= & e^{-t \mathcal{L}}\left(x_{0}-\mathcal{L}^{-1} F(0)\right)+e^{-t \mathcal{L}} \mathcal{L}^{-1}(F(0)-F(t)) \\
& +\int_{0}^{t} e^{-(t-s) \mathcal{L}}(F(s)-F(t)) d s+\mathcal{L}^{-1} F(t) .
\end{aligned}
$$

The first term is in $C^{\alpha}\left(I, X^{++}\right)$by the compatibility condition; for the other terms we can use the special semigroup estimates. The compatibility condition in $t$ is proved in a similar manner.

We complete our analysis of the nonstationary equation with a remark about the size of the space $D_{\mathcal{L}, \alpha}$. The preceding theorem shows that it contains all functions $(u(t), \eta(t))$ that can be reached with solutions starting from 0 . It furthermore contains all smooth functions with appropriate boundary data. By writing $e^{-t \mathcal{L}_{x_{0}}-x_{0}}$ as a complex integral one proves, for $X=X^{r}$,

$$
\left\{x_{0} \in \tilde{X} \mid \mathcal{L} x_{0} \in \tilde{X}^{r+4}\right\} \subset D_{\mathcal{L}, \alpha} .
$$

Concerning the time-dependent problem, we finally remark that the equations for the center of mass and rotations can now be solved with one additional integration.

4. The nonlinear liquid-drop equation. We now consider the full free boundary problem. We will need high orders of regularity and set $X=X^{2}, X^{++}=X^{4}$. In this section we do not impose the conditions of vanishing momentum and vanishing angular momentum and extend $\mathcal{L}^{-1}$ trivially.

The transformation of the equations (1)-(6) to a fixed domain is done in the standard way, as in Beale [2]. Using the operator $\mathcal{L}$, the transformed equations read

$$
\partial_{t}\left(\begin{array}{l}
v \\
\eta
\end{array}\right)+\mathcal{L}\left(\begin{array}{l}
v \\
\eta
\end{array}\right)=\left(\begin{array}{c}
F(v, \eta) \\
0
\end{array}\right)
$$

with the boundary condition

$$
\tau_{i} \cdot S_{v} \cdot n=G_{i}(v, \eta)
$$

In the case $r \geq 1$,

$$
\begin{gathered}
F: X^{r+2} \rightarrow H^{r}\left(B^{3}\right)^{3}, F(0,0)=0, D F \text { exists, and } D F(0,0)=0 \\
G: X^{r+2} \rightarrow H^{r+1-\frac{1}{2}}\left(S^{2}\right)^{2}, G(0,0)=0, D G \text { exists, and } D G(0,0)=0 .
\end{gathered}
$$

We solve the time-dependent problem by means of an iteration. The boundary condition (15) is satisfied with the help of a function $\Phi$. 
Definition 4.1. For functions $g_{i}$ we define a vector field $\Phi(g): B^{3} \rightarrow \mathbb{R}^{3}$ which has the correct boundary values. With the help of the Stokes operator $A$ we define $\Phi(g): B^{3} \rightarrow \mathbb{R}^{3}$ as the solution of

$$
\begin{aligned}
A \Phi(g) & =0 \text { in } B^{3}, \\
\Phi_{n}(g) & =0 \text { on } S^{2}, \\
\tau_{i} \cdot S_{\Phi(g)} \cdot n & =g_{i} \text { on } S^{2} .
\end{aligned}
$$

We consider new variables, namely,

$$
\tilde{x}=x-\left(\begin{array}{c}
\Phi(G(x)) \\
0
\end{array}\right)
$$

with inverse $x=\xi(\tilde{x})$. The boundary condition (15) is satisfied if we construct $\tilde{x} \in \tilde{X}$. In the $\tilde{x}$-variable the equations read

$$
\begin{aligned}
\left(\partial_{t}+\mathcal{L}\right) \tilde{x} & =\left(\begin{array}{c}
\tilde{F}(\tilde{x}) \\
0
\end{array}\right):=\left(\begin{array}{c}
F \circ \xi(\tilde{x}) \\
0
\end{array}\right)-\left(\partial_{t}+\mathcal{L}\right)\left(\begin{array}{c}
\Phi \circ G \circ \xi(\tilde{x}) \\
0
\end{array}\right), \\
\tilde{x}(0) & =\xi^{-1}\left(x_{0}\right)=x_{0}-\left(\Phi \circ G\left(x_{0}\right), 0\right) .
\end{aligned}
$$

We remark that we have a vanishing second component in the right-hand side.

In the following, we impose the conditions that initial values satisfy (5) and that the formal time derivative at 0 has the appropriate regularity.

DeFINITION 4.2. $x_{0}$ satisfies the nonlinear compatibility conditions in $X^{++}$if

$$
\begin{aligned}
\tilde{x}_{0} & :=x_{0}-\left(\begin{array}{c}
\Phi\left(G\left(x_{0}\right)\right) \\
0
\end{array}\right) \in \tilde{X}^{++}, \\
z & :=\tilde{x}_{0}-\mathcal{L}^{-1}\left(\tilde{F}\left(\tilde{x}_{0}\right), 0\right) \in D_{\mathcal{L}, \alpha} .
\end{aligned}
$$

Before we state the theorem of local existence and uniqueness, we investigate the compatibility conditions in more detail. The following proposition states that the permitted small initial values form a Banach manifold. We will use this fact to prove a Hopf bifurcation; the idea is taken from Koch [9].

Proposition 4.3. There exists $U=B_{\varepsilon}(0) \subset D_{\mathcal{L}, \alpha}$ and a mapping $\zeta: U \rightarrow X^{++}$ such that every $x_{0}=\zeta(z)$ satisfies the nonlinear compatibility conditions with small norms. We denote the manifold $\zeta(U)$ by $\mathcal{M} . \zeta$ can be constructed with $D \zeta(0)=i d$.

Proof. We only have to invert the equation (19) with $x_{0} \in \tilde{X}^{++}$. We use the contraction mapping principle for the map

$$
\tilde{X}^{++} \ni \tilde{x}_{0} \mapsto z+\mathcal{L}^{-1}\left(\tilde{F}\left(\tilde{x}_{0}\right), 0\right) \in \tilde{X}^{++} .
$$

This yields the fixed point $\tilde{x}_{0}=\tilde{\zeta}(z)$. We define $\zeta:=\xi \circ \tilde{\zeta}$.

TheOREM 4.4. According to small initial values $x_{0}$ satisfying the compatibility conditions, i.e.,

$$
x_{0}=\zeta(z) \in \mathcal{M}, \quad\|z\|_{K, \alpha} \text { small },
$$

there exists a unique small solution of the nonlinear liquid-drop equation in

$$
Z^{++}=C^{1, \alpha}\left(I, X^{2}\right) \cap C^{\alpha}\left(I, X^{4}\right) .
$$

As a map on the Banach manifold $\mathcal{M}$, the flow is differentiable. 
Proof. We construct the solution with the help of an iteration map $T: \tilde{Z}^{++} \rightarrow$ $\tilde{Z}^{++}$. Let $(v, \sigma) \in \tilde{Z}^{++}$be given. We define $\tilde{f}=\tilde{F}(v, \sigma)$ and solve

$$
\begin{gathered}
\left(\partial_{t}+\mathcal{L}\right) \tilde{x}=(\tilde{f}, 0), \\
\tilde{x}(0)=x_{0}-\left(\Phi \circ G\left(x_{0}\right), 0\right)=\tilde{\zeta}(z)
\end{gathered}
$$

for $\tilde{x} \in \tilde{Z}^{++}$with the help of Theorem 3.4. Because of $D F(0,0)=0$ and $D G(0,0)=0$, the solution operator is contracting in a small ball $B_{\varepsilon}(0) \subset Z^{++}$and there exists a unique fixed point $\tilde{x}$. The function $\xi(\tilde{x})$ is a solution of the nonlinear equation.

We have to take care that in the iteration the right-hand side is contained in the function space. The condition of vanishing divergence can be assured with the usual projection. This yields an additional pressure that vanishes at the boundary.

It remains to show the differentiability of the flow $x_{0} \mapsto x(t)$ on the manifold, i.e., the differentiability of

$$
\begin{array}{r}
\Phi_{t}: \bar{x}_{0} \mapsto \zeta^{-1} x(t)=x(t)-\mathcal{L}^{-1} \tilde{F}(x(t)) \quad \text { with } \\
\bar{x}_{0}=\zeta^{-1}\left(x_{0}\right)=x_{0}-\mathcal{L}^{-1} \tilde{F}\left(x_{0}\right)=\bar{x}_{0}
\end{array}
$$

We omit the straightforward calculation, proving that the derivative of $\Phi_{t}$ can be written as

$$
D \Phi_{t}: \bar{w}_{0} \mapsto\left(i d-\left.\mathcal{L}^{-1} \circ D \tilde{F}\right|_{x(t)}\right) w(t)
$$

where $w($.$) solves$

$$
\begin{gathered}
\left(\partial_{t}+\mathcal{L}\right) w(.)=\left.D \tilde{F}\right|_{x(.)} \cdot w(.), \\
w(0)=w_{0}=\left(i d-\left.\mathcal{L}^{-1} \circ D \tilde{F}\right|_{x(t)}\right)^{-1} \bar{w}_{0} .
\end{gathered}
$$

5. The spectrum of $\mathcal{L}$. The eigenvalues of $\mathcal{L}$ can be calculated explicitly for $\beta=0$. We investigate the movement of the eigenvalues in the complex plane as $\beta \rightarrow \infty$. We prove the qualitative behavior that has been observed numerically (compare [3]).

We will make fundamental use of the fact that the liquid-drop problem has an $O(3)$ symmetry, in other words, that $\mathcal{L}$ is $O(3)$-equivariant. The group action will be denoted by ${ }^{*}$. We use the decomposition $X=\bigoplus X_{n}$, and we describe the spectrum on $X_{n}$ for $n \geq 2$.

The $n$th eigenspace of $\Delta_{B}, \Psi_{n}$ has the standard basis $\left(\psi_{n,-n}, \ldots, \psi_{n, n}\right)$ with

$$
\psi_{n, k}(\theta, \varphi)=P_{n, k}(\cos (\theta)) e^{i k \varphi} .
$$

The function $\Phi_{0}:=\psi_{n, 0}$ has an isotropy subgroup $\Gamma$ isomorphic to $O(2)$. Any function $\psi: S^{2} \rightarrow \mathbb{R}$ can be $\Gamma$-symmetrized by

$$
\bar{\psi}(\xi):=f_{\Gamma} \gamma * \psi(\xi) d \gamma .
$$

The same can be done with functions $v: B^{3} \rightarrow \mathbb{R}^{3}$.

In this and the following section we consider only eigenfunctions $(u, \eta) \in X_{n}$ with $\eta=\Phi_{0}$. This is no restriction since every eigenfunction can be projected and symmetrized such that the second component is a multiple of $\Phi_{0}$. 
We make constant use of the following observation: given an eigenvalue $z$ of $\mathcal{L}$, we can construct the eigenfunction $\left(u, \Phi_{0}\right)$ as the solution of a Stokes problem.

Definition 5.1. By $A_{N}$ we denote the Stokes operator in the space of functions with vanishing normal component at the boundary. The eigenvalues of $A_{N}$ are denoted by $\left\{\kappa_{j}\right\}_{j \in \mathbb{N}}$. The corresponding eigenfunctions with symmetry $\Gamma$ are denoted by $\left\{u_{j}\right\}_{j \in \mathbb{N}}$; and the pressure, by $\left\{p_{j}\right\}_{j \in \mathbb{N}}$. Their signs are determined in (24).

For $z \in \mathbb{C} \backslash\left\{\kappa_{j} \mid j \in \mathbb{N}\right\}$ we define $(\tilde{u}(z), \tilde{p}(z))$ as the unique solution of the system

$$
\begin{aligned}
z \tilde{u}(z)+\nu \Delta \tilde{u}(z)-\nabla \tilde{p}(z) & =0, \\
\nabla \cdot \tilde{u}(z) & =0, \\
\left.\tau \cdot S_{\tilde{u}(z)}\right|_{\partial} \cdot n & =0, \\
\left.\tilde{u}_{n}(z)\right|_{\partial} & =-z \Phi_{0} .
\end{aligned}
$$

The solution has the same symmetry as $\Phi_{0}$, i.e., $\Gamma$. In particular, $\left.\left(\tilde{p}(z)-2 \nu S_{\tilde{u}(z)}^{n}\right)\right|_{\partial}$ has the symmetry $\Gamma$ and is a multiple of $\Phi_{0}$. We define $\tilde{r}(z) \in \mathbb{C}$ by

$$
\left.\left(\tilde{p}(z)-2 \nu n \cdot S_{\tilde{u}(z)} \cdot n\right)\right|_{\partial}=: \tilde{r}(z) \Phi_{0} .
$$

We remark that $z \in \mathbb{R}$ implies $\tilde{r}(z) \in \mathbb{R}$.

Any $z \in \mathbb{C} \backslash\left\{\kappa_{j} \mid j \in \mathbb{N}\right\}$ is an eigenvalue of $\mathcal{L}$ with eigenfunction $\left(\tilde{u}(z), \Phi_{0}\right)$ if it satisfies

$$
\tilde{r}(z)=\Lambda_{k} \beta .
$$

We remark that the functions $\tilde{u}(z)$ and $\tilde{p}(z)$ can be computed explicitly in terms of Bessel functions; this can be used to analyze the function $\tilde{r}(z)$ numerically. In the following, $\|$.$\| denotes the L^{2}$-norm.

Proposition 5.2 (properties of $\tilde{u}(z)$ ). $\tilde{u}(z)$ is a differentiable family of functions for $z \in \mathbb{C} \backslash\left\{\kappa_{j} \mid j \in \mathbb{N}\right\}$. In $\kappa_{j}$,

$$
\|\tilde{u}(z)\| \rightarrow \infty \quad \text { for } \quad z \rightarrow \kappa_{j} .
$$

The rescaled functions approximate the Stokes eigenfunctions

$$
u_{j}=\lim _{\mathbb{R} \ni z \nearrow \kappa_{j}} \frac{\tilde{u}(z)}{\|\tilde{u}(z)\|}=\lim _{\mathbb{R} \ni z \searrow \kappa_{j}} \frac{-\tilde{u}(z)}{\|\tilde{u}(z)\|} .
$$

Furthermore,

$$
\|\tilde{u}(z)\| \rightarrow \infty \quad \text { for } \quad|z| \rightarrow \infty .
$$

Proof. We define a family of functions $(u(z), p(z))$ which depends smoothly on $z$ in a neighborhood of $\kappa_{j}$ by solving

$$
\begin{aligned}
z u(z)+\nu \Delta u(z)-\nabla p(z) & =0 \\
\nabla \cdot u & =0 \\
\left.\tau \cdot S_{u(z)} \cdot n\right|_{\partial}=0,\left.\quad\left(p(z)-2 \nu S_{u(z)}^{n}\right)\right|_{\partial} & =\Phi_{0} .
\end{aligned}
$$

The solution is unique; therefore, $u\left(\kappa_{j}\right)$ is a multiple of $u_{j}$. With the notation

$$
\left.u_{n}(z)\right|_{\partial}=: s(z) \Phi_{0},
$$


$s($.$) is continuous and s\left(\kappa_{j}\right)=0$. (23) is proved by observing

$$
\tilde{u}(z)=\frac{-z}{s(z)} u(z) .
$$

(24) is proved by showing that the function $\left.s()\right|_{.\mathbb{R}}$ changes sign in $\kappa_{j}$.

Assume $\partial_{z} s\left(\kappa_{j}\right)=0$. Then $v:=\partial_{z} u\left(\kappa_{j}\right), q:=\partial_{z} p\left(\kappa_{j}\right)$ satisfies the boundary conditions

$$
\left.\tau \cdot S_{v}\right|_{\partial} \cdot n=0,\left.\quad v_{n}\right|_{\partial}=0,\left.\quad\left(q-2 \nu S_{v}^{n}\right)\right|_{\partial}=0
$$

Additionally,

$$
\kappa_{j} v+\nu \Delta v-\nabla q=-u\left(\kappa_{j}\right)
$$

Multiplying with $u\left(\kappa_{j}\right)$ and integrating yields $0=-\left\|u\left(\kappa_{j}\right)\right\|^{2}$ - a contradiction.

(25) can be proved directly. As the solution of the Stokes system (20), $\tilde{u}$ satisfies an estimate

$$
\|\tilde{u}(z)\|_{H^{2}} \leq C_{S}\left\{|z|\|\tilde{u}(z)\|_{L^{2}}+|z|\left\|\Phi_{0}\right\|_{H^{3 / 2}\left(S^{2}\right)}\right\} .
$$

We now use $\left.\tilde{u}_{n}(z)\right|_{\partial}=-z \Phi_{0}$, a trace formula, and an interpolation to calculate

$$
\begin{aligned}
|z|^{2}\left\|\Phi_{0}\right\|_{L^{2}\left(S^{2}\right)}^{2} & =\left\|\left.\tilde{u}_{n}(z)\right|_{\partial}\right\|_{L^{2}\left(S^{2}\right)}^{2} \\
& \leq C_{T}\|\tilde{u}(z)\|_{H^{1}}^{2} \\
& \leq C_{T} C_{c}\|\tilde{u}(z)\|_{L^{2}}\|\tilde{u}(z)\|_{H^{2}} \\
& \leq C_{T} C_{c} C_{S}\|\tilde{u}(z)\|_{L^{2}}\left\{|z|\|\tilde{u}(z)\|_{L^{2}}+|z|\left\|\Phi_{0}\right\|_{H^{3 / 2}\left(S^{2}\right)}\right\}
\end{aligned}
$$

This yields $\|\tilde{u}(z)\|_{L^{2}}^{2} \geq$ const $\cdot|z|$, and the proposition is proved.

PROPOSITION 5.3 (properties of $\tilde{r}(z)$ ). The function $\tilde{r}(z)$ satisfies

$$
\begin{aligned}
& \tilde{r}(z) \rightarrow 0 \text { for } \mathbb{R} \ni z \searrow 0, \\
& \tilde{r}(z) \rightarrow-\infty \text { for } \mathbb{R} \ni z \nearrow \kappa_{j}, \\
& \tilde{r}(z) \rightarrow+\infty \text { for } \mathbb{R} \ni z \searrow \kappa_{j} .
\end{aligned}
$$

$\tilde{r}(z)$ is positive for small $z>0$,

$$
\partial_{z} \tilde{r}(0)>0 .
$$

Between $\kappa_{j}$ and $\kappa_{j+1}$, there is at most one turning point. Critical values of $\tilde{r}(z)$ are positive.

Proof. The assertion of (26) for $z \rightarrow 0$ is trivial. $u_{j}$ satisfies

$$
u_{j}=\lim _{z \nearrow \kappa_{j}} \frac{\tilde{u}(z)}{\|\tilde{u}(z)\|} .
$$

We test the eigenvalue equation of $u_{j}$ with $v:=\frac{\tilde{u}\left(\kappa_{j}-\varepsilon\right)}{\left\|\tilde{u}\left(\kappa_{j}-\varepsilon\right)\right\|}$ to get

$$
\begin{aligned}
0 & =\left\langle\left(\kappa_{j}-A\right) u_{j}, v\right\rangle \\
& =\left\langle u_{j},\left(\kappa_{j}-A\right) v\right\rangle-\left.\left.\int_{S}\left(p_{j}-2 \nu S_{u_{j}}^{n}\right)\right|_{\partial} v_{n}\right|_{\partial} \\
& =\varepsilon\left\langle u_{j}, v\right\rangle-\left.\left.\int_{S}\left(p_{j}-2 \nu S_{u_{j}}^{n}\right)\right|_{\partial} v_{n}\right|_{\partial} .
\end{aligned}
$$


By (24) the first term is positive for small $|\varepsilon|$; the second has the sign of $\tilde{r}\left(\kappa_{j}-|\varepsilon|\right)$.

To prove (27) we consider the functions $v:=\partial_{z} \tilde{u}(0), q=\partial_{z} \tilde{p}(0)$. They solve

$$
\begin{gathered}
\nu \Delta v-\nabla q=0, \\
\left.\tau \cdot S_{v}\right|_{\partial} \cdot n=0,\left.\quad v_{n}\right|_{\partial}=-\Phi_{0},\left.\quad\left(q-2 \nu S_{v}^{n}\right)\right|_{\partial}=\partial_{z} \tilde{r}(0) \Phi_{0} .
\end{gathered}
$$

Multiplying this equation with $v$ yields

$$
-\int_{B^{3}}\left|S_{v}\right|^{2}+\partial_{z} \tilde{r}(0)\left\|\Phi_{0}\right\|^{2}=0
$$

which proves (27).

We claim that turning points of $\tilde{r}(z), z \in \mathbb{R}$, are the critical points of $\|\tilde{u}(z)\|$, $z \in \mathbb{R}$. We consider the functions $v(z):=\partial_{z} \tilde{u}(z), q(z):=\partial_{z} \tilde{p}(z)$, which solve

$$
\begin{aligned}
& \tilde{u}(z)+z v(z)+\nu \Delta v(z)-\nabla q(z)=0, \\
& \left.\tau \cdot S_{v(z)}\right|_{\partial} \cdot n=0,\left.\quad v_{n}(z)\right|_{\partial}=-\Phi_{0}, \\
& \left.\left(q(z)-2 \nu S_{v(z)}^{n}\right)\right|_{\partial}=\partial_{z} \tilde{r}(z) \Phi_{0} .
\end{aligned}
$$

Multiplying (28) by $\tilde{u}(z)$ yields

$$
\|\tilde{u}(z)\|^{2}+z \partial_{z} \tilde{r}(z)\left\|\Phi_{0}\right\|^{2}-\tilde{r}(z)\left\|\Phi_{0}\right\|^{2}=0,
$$

and differentiating gives

$$
\partial_{z}\|\tilde{u}(z)\|^{2}+z \partial_{z}^{2} \tilde{r}(z)\left\|\Phi_{0}\right\|^{2}=0 .
$$

Consider $w:=\partial_{z}^{2} \tilde{u}\left(z_{0}\right), r:=\partial_{z}^{2} \tilde{p}\left(z_{0}\right)$, which solve

$$
\begin{aligned}
& 2 v+\kappa_{j} w+\nu \Delta w-\nabla r=0, \\
& \left.\tau \cdot S_{w}\right|_{\partial} \cdot n=0,\left.\quad w_{n}\right|_{\partial}=0, \\
& \left.\left(r-2 \nu S_{w}^{n}\right)\right|_{\partial}=\partial_{z}^{2} \tilde{r}\left(z_{0}\right) \Phi_{0} .
\end{aligned}
$$

Multiplying (31) with $v$ yields

$$
2\|v\|^{2}-\left\langle\tilde{u}\left(z_{0}\right), w\right\rangle+\partial_{z}^{2} \tilde{r}\left(z_{0}\right)\left\|\Phi_{0}\right\|^{2}=0 .
$$

Assume there is more than one point with $\partial_{z}^{2} \tilde{r}=0$. By (30) they coincide with critical points of $\|\tilde{u}(z)\|$. At least one of them satisfies $\partial_{z}^{2}\|\tilde{u}(z)\|^{2} \leq 0$. This contradicts equation (32), which implies

$$
\partial_{z}^{2}\|\tilde{u}(z)\|^{2}=2\left\langle\tilde{u}\left(z_{0}\right), w\right\rangle+2\|v\|^{2}=6\|v\|^{2}>0 .
$$

The last assertion of the proposition follows from (29).

We denote the Stokes operator with boundary condition $\left.\left(p-2 \nu S_{u}^{n}\right)\right|_{\partial}=0$ by $A_{S}$ and the eigenvalues of $A_{S}$ by $\left\{\rho_{j}\right\}_{j \in \mathbb{N}}$.

Theorem 5.4 (the spectrum of $\mathcal{L}$ in dependence of $\beta$ ). It holds that

$$
\rho_{0}<\kappa_{0}<\rho_{1}<\cdots<\rho_{j}<\kappa_{j}<\cdots .
$$

For $\beta=0$ all the eigenvalues of $\mathcal{L}_{\beta}$ are real. Denoting them by $\left(\mu_{j}\right)_{j \in \mathbb{N}}$,

$$
\mu_{0}=0, \quad \mu_{j+1}=\rho_{j} .
$$


For small $\beta$ the eigenvalues stay real. With increasing $\beta$ the first eigenvalue moves to the right while the other eigenvalues move to the left. For some $\beta_{0}>0$ the first two eigenvalues merge and leave the real axis.

Given a radius $k$ there exists $\beta_{k}>0$ such that for $\beta>\beta_{k}$ the following is true. The norm of nonreal eigenvalues of $\mathcal{L}_{\beta}$ is larger than $k$. Every interval $\left[\kappa_{j}, \kappa_{j+1}\right]$ with $\kappa_{j+1}<k$ contains one and only one eigenvalue $\mu(\beta)$ of $\mathcal{L}_{\beta}$. This eigenvalue satisfies

$$
\mu(\beta) \searrow \kappa_{j} \text { for } \beta \rightarrow \infty .
$$

Proof. The numbers $\rho_{j}, j \in \mathbb{N}$ are the zeros of $\tilde{r}(z)$. The shape of $\tilde{r}$ implies the assertion on the position of the Stokes eigenvalues. For $\beta=0$ we can compute a complete set of eigenfunctions in $X_{k}: \mu_{0}=0$ with eigenfunction $\left(0, \Phi_{0}\right)$ and $\mu_{j+1}=\rho_{j}$ with eigenfunctions $\left(\tilde{u}\left(\rho_{j}\right), \Phi_{0}\right)$. By the shape of $\tilde{r}(z)$ and $(22)$ the first two eigenvalues meet at the maximum of $\tilde{r}$ and must leave the real axis. Eigenfunctions $\left(u(\beta), \Phi_{0}\right)$ of $\mathcal{L}_{\beta}$ with nonreal eigenvalues $\mu(\beta)$ satisfy the energy equality

$$
\|u\|_{L^{2}}=\beta \Lambda_{k}\left\|\Phi_{0}\right\|_{L^{2}\left(S^{2}\right)}^{2} .
$$

Therefore, nonreal eigenvalues cannot stay bounded for $\beta \rightarrow \infty$. The shape of $\tilde{r}$ together with (22) prescribes the movement of the real eigenvalues as stated. The theorem is proved.

REMARK 5.5. Eigenvalues leave the real axis with an infinite speed. The qualitative shape of $\tilde{r}_{\nu}(z)$ is independent of the viscosity $\nu$ :

$$
\tilde{r}_{\alpha \nu}(\alpha z)=\alpha^{2} \tilde{r}_{\nu}(z) \text {. }
$$

Proof. Eigenvalues leave the real axis in a critical point $z_{0}$ of $\tilde{r}$, an analytic function in $\mathbb{C} \backslash\left\{\kappa_{j} \mid j \in \mathbb{N}\right\}$. It holds that $\frac{\partial \operatorname{Re}\left(\tilde{r}\left(z_{0}\right)\right)}{\partial \operatorname{Im} z}=0$, and (22) implies

$$
\Lambda_{k}=\frac{\partial \operatorname{Re}(\tilde{r}(z(\beta)))}{\partial \beta}=\frac{\partial \operatorname{Re}(\tilde{r}(z))}{\partial z} \frac{\partial z}{\partial \beta} .
$$

The speed of $z(\beta)$ gets infinite.

The statement on the shape of $\tilde{r}_{\nu}(z)$ is proved by multiplying the equation for $\tilde{u}$ by $\alpha^{2}$ :

$$
\begin{aligned}
(\alpha z)(\alpha u)+(\alpha \nu) \Delta(\alpha u)-\nabla\left(\alpha^{2} p\right) & =0 \\
\left.(\alpha u)_{n}\right|_{\partial} & =-(\alpha z) \Phi_{0}, \\
\left.\left(\alpha^{2} p-2(\alpha \nu) S_{\alpha u}^{n}\right)\right|_{\partial} & =\alpha^{2} \tilde{r}_{\nu}(z) .
\end{aligned}
$$

By definition of $\tilde{r}$ the last line coincides with $\tilde{r}_{\alpha \nu}(\alpha z)$.

6. A Hopf bifurcation for liquid drops. In this section we show how our analysis can be used to study the effect of an exterior force. In the previous section, we achieved a complete picture of the spectrum of $\mathcal{L}$. On a fixed subspace $X_{k}$, the spectrum consists of a countable number of eigenvalues that correspond to eigenvalues of the Stokes operator and two additional eigenvalues (interfacial eigenvalues) that are real for small surface tension and nonreal for large surface tension.

Starting from this situation, a Hopf bifurcation can occur if an exterior force moves the additional eigenvalues across the imaginary axis. We prove this behavior in the case of a force that preserves symmetry. A more physical force will not preserve symmetry; we study that case in section 7 . 
In this section we assume that the force acts on the surface and that its strength depends linearly on the position and the speed of the boundary. The symmetric force has only two parts; the one proportional to $\eta$ acts as the surface tension, and we restrict our analysis to a force proportional to $\partial_{t} \eta(x, t)=\left.u_{n}\right|_{\partial}$. We introduce the real number $\lambda$ for its strength and have the boundary condition

$$
\left.\left(p-\nu S_{u}^{n}\right)\right|_{\partial}+\beta \Delta \eta=\left.\lambda u_{n}\right|_{\partial} .
$$

We write the linear equations again as

$$
\frac{d}{d t} x+\mathcal{L}_{\lambda} x=0, \quad x \in \tilde{X}
$$

now with the operator

$$
\mathcal{L}_{\lambda}\left(\begin{array}{c}
u \\
\eta
\end{array}\right):=\left(\begin{array}{c}
-\nu \Delta u+\nabla \mathcal{H}\left(2 \nu S_{u}^{n}\right)-\nabla \mathcal{H}(\beta \Delta \eta)-\nabla \mathcal{H}\left(\left.\lambda u_{n}\right|_{\partial}\right) \\
-\left.u_{n}\right|_{\partial}
\end{array}\right) .
$$

This operator is a lower order perturbation of $\mathcal{L}$; its spectrum consists of eigenvalues and we have the local existence results as before. The following analogue of Lemma 2.4 holds.

Lemma 6.1. Let $(u, \eta) \in \tilde{X}^{2}$ be an eigenvector of $\mathcal{L}_{\lambda}$ with eigenvalue $\mu$. Then

$$
\begin{aligned}
& \operatorname{Re}(\mu)\|(u, \eta)\|_{E}^{2}=2 \nu\left(\int_{B}\left|S_{u}\right|^{2}\right)-\lambda|\mu|^{2}\|\eta\|_{L^{2}\left(S^{2}\right)}^{2}, \\
& \operatorname{Im}(\mu)\|(u, \eta)\|_{E}^{2}=2 \beta \operatorname{Im}\left(\left.\int_{S} u_{n}\right|_{\partial} \Delta \bar{\eta}\right) .
\end{aligned}
$$

In the case of nonreal eigenvalues, $\operatorname{Im}(\mu) \neq 0$, the following energy equality holds:

$$
\|u\|_{E}^{2}=\|\eta\|_{E}^{2}=\frac{1}{2}\|(u, \eta)\|_{E}^{2} .
$$

Proof. This lemma is proved as Lemma 2.4.

We again want to get a global picture of the position of eigenvalues, now in dependence of the parameter $\lambda$. There are two important differences from the previous section:

- the eigenvalues may have a negative real part and

- the energy equality for eigenvectors implies that nonreal eigenvalues are bounded independent of $\lambda$.

For any $z \in \mathbb{R}_{+} \backslash\left\{\kappa_{j} \mid j \in \mathbb{N}\right\}$ we have defined the function $\tilde{u}(z)$. The pair $\left(\tilde{u}(z), \Phi_{0}\right)$ is an eigenfunction of $\mathcal{L}_{\tilde{\lambda}}$, with $\tilde{\lambda}(z)$ defined by

$$
\tilde{r}(z) \Phi_{0}=\left.\left(\tilde{p}(z)-2 \nu S_{\tilde{u}(z)}^{n}\right)\right|_{\partial}=\beta \Lambda_{k} \Phi_{0}+\tilde{\lambda}(z) z \Phi_{0} .
$$

Proposition 6.2 (properties of $\tilde{\lambda}(z)$ ). There exists a constant $\lambda_{0}>0$ such that

$$
\begin{aligned}
& |\lambda|>\lambda_{0} \Rightarrow \text { all eigenvalues of } \mathcal{L}_{\lambda} \text { are real, } \\
& \tilde{\lambda}(z) \rightarrow-\infty \text { for } \mathbb{R} \ni z \rightarrow 0, \\
& \tilde{\lambda}(z) \rightarrow-\infty \text { for } \mathbb{R} \ni z \nearrow \kappa_{j}, \\
& \tilde{\lambda}(z) \rightarrow+\infty \text { for } \mathbb{R} \ni z \searrow \kappa_{j} .
\end{aligned}
$$


Proof. Equation (33) implies, for nonreal eigenvalues, $|\lambda| \rightarrow \infty \Rightarrow \int\left|S_{u}\right|^{2} \rightarrow \infty$ or $|\mu| \rightarrow \infty$ or $|\mu| \rightarrow 0$. Therefore, $|\mu| \rightarrow \infty$ or $|\mu| \rightarrow 0$. (25) implies $\|u\| \rightarrow \infty$ or $\|u\| \rightarrow 0$. This contradicts (34). (37)-(39) follow from $\tilde{\lambda}(z)={ }_{z}^{1}\left(\tilde{r}(z)-\beta \Lambda_{k}\right)$.

Theorem 6.3 (the spectrum of $\mathcal{L}$ in dependence of $\lambda$ ). ${ }^{z}$ For $\lambda<-\lambda_{0}$, all eigenvalues of $\mathcal{L}_{\lambda}$ are real. Denoting the ordered sequence of them by $\left(\mu_{j}(\lambda)\right)_{j \in \mathbb{N}}$, they satisfy

$$
\begin{aligned}
& 0<\mu_{0}(\lambda)<\mu_{1}(\lambda)<\kappa_{0}, \quad \kappa_{j}<\mu_{j+2}<\kappa_{j+1}, \\
& \mu_{0}(\lambda) \searrow 0 \text { for } \lambda \rightarrow-\infty \quad \text { and } \\
& \mu_{j+2}(\lambda) \nearrow \kappa_{j+1} \text { for } \lambda \rightarrow-\infty .
\end{aligned}
$$

For $\lambda>\lambda_{0}$ the ordered eigenvalues satisfy

$$
\begin{aligned}
& \mu_{0}(\lambda), \mu_{1}(\lambda)<0, \quad \kappa_{j}<\mu_{j+2}<\kappa_{j+1}, \\
& \mu_{j+2}(\lambda) \searrow \kappa_{j} \text { for } \lambda \rightarrow \infty .
\end{aligned}
$$

In a point $\bar{\lambda} \in\left[-\lambda_{0}, \lambda_{0}\right]$, a pair of conjugate complex eigenvalues crosses the imaginary axis transversally. The imaginary axis can be crossed only from right to left.

Proof. Proposition 6.2 implies the assertion for the position of positive real eigenvalues for $|\lambda| \rightarrow \infty$. We have to prove the existence of the pair of negative eigenvalues. We do this by counting eigenvalues. (25), together with the energy equality (34), implies that nonreal eigenvalues are bounded independent of $\lambda$. Let $\kappa_{J}$ be larger than this bound. We restrict ourselves to the $J+2$ eigenvalues of $\mathcal{L}_{-\lambda_{0}}$ with norm smaller than $\kappa_{J}$.

For $\lambda \rightarrow+\infty$ (and, in particular, $\lambda>\lambda_{0}$ ) there are $J$ eigenvalues with positive real part and norm less than $\kappa_{J}$. The two remaining eigenvalues must be negative.

To count the eigenvalues, we used the fact that geometric and algebraic multiplicity coincide for $|\lambda| \rightarrow \infty$. We now prove this fact.

Assumption. There exist normed functions $(u, \eta),(v, \sigma)$ satisfying

$$
\mathcal{L}\left(\begin{array}{c}
u \\
\eta
\end{array}\right)=\mu\left(\begin{array}{c}
u \\
\eta
\end{array}\right), \mathcal{L}\left(\begin{array}{c}
v \\
\sigma
\end{array}\right)=\mu\left(\begin{array}{c}
v \\
\sigma
\end{array}\right)+\alpha\left(\begin{array}{l}
u \\
\eta
\end{array}\right), v \perp u .
$$

Let $p$ denote the pressure function corresponding to $u$. We know $(u, \eta) \rightarrow\left(u_{j}, 0\right)$ for $\lambda \rightarrow \infty$. We define $\left(v_{0}, \sigma_{0}\right)=\lim _{\lambda \rightarrow \infty}(v, \sigma)$. It holds that

$$
\begin{aligned}
-\nu \Delta v+\nabla \mathcal{H}\left(2 \nu S_{v}^{n}\right)-\nabla \mathcal{H}\left(\beta \Delta \sigma+\left.\lambda v_{n}\right|_{\partial}\right) & =\mu v+\alpha u \\
-v_{n} & =\mu \sigma+\alpha \eta .
\end{aligned}
$$

To prove that $\alpha$ is bounded we multiply (40) by $u_{j}$ and integrate to get

$$
\begin{aligned}
\alpha\left\langle u, u_{j}\right\rangle & =\left\langle-\mu v-\nu \Delta v+\nabla \mathcal{H}\left(2 \nu S_{v}^{n}\right)-\nabla \mathcal{H}\left(\beta \Delta \sigma+\left.\lambda v_{n}\right|_{\partial}\right), u_{j}\right\rangle \\
& =\left\langle v,\left(A_{N}-\mu\right) u_{j}\right\rangle+\int_{S^{2}} v_{n}\left(p_{j}-2 \nu S_{u_{j}}^{n}\right) \\
& =\left(\kappa_{j}-\mu\right)\left\langle v, u_{j}\right\rangle+\int_{S^{2}} v_{n}\left(p_{j}-2 \nu S_{u_{j}}^{n}\right) .
\end{aligned}
$$

Using $\eta \rightarrow 0$, this implies that $\alpha$ is bounded. Equation (40) implies $\left(v_{0}\right)_{n}=0$, and we get, from equation (41), $\sigma_{0}=0$. With $\alpha_{0}:=\lim \alpha$,

$$
\left(A_{N}-\kappa_{j}\right) v_{0}=\alpha_{0} u_{j}
$$


a contradiction to the simplicity of the Stokes eigenvalues.

We now derive explicit equations for the velocity of eigenvalues. We consider a differentiable family of eigenvalues $\mu(\lambda) \in \mathbb{C} \backslash \mathbb{R}$ with eigenfunctions $\left(u(\lambda), \Phi_{0}\right)$.

We first need an equation for $\operatorname{Im}\left\langle u, \partial_{\lambda} u\right\rangle$. We multiply the eigenvalue equation by $\partial_{\lambda} u$ to get, with the help of $\left.\partial_{\lambda} u_{n}\right|_{\partial}=-\partial_{\lambda} \mu(\lambda) \Phi_{0}$,

$$
\left\langle\mu(\lambda) u(\lambda), \partial_{\lambda} u(\lambda)\right\rangle=2 \nu \int_{B^{3}} S_{u}: S_{\partial_{\lambda} \bar{u}}-\left(\beta \Lambda_{k}+\lambda \mu(\lambda)\right) \partial_{\lambda} \bar{\mu}(\lambda)\left\|\Phi_{0}\right\|_{L^{2}}^{2} .
$$

Taking the real part and using Lemma 6.1 yields

$$
\begin{aligned}
\operatorname{Re}\left\langle\mu u, \partial_{\lambda} u\right\rangle= & \frac{1}{2} \partial_{\lambda}\left(2 \nu \int_{B^{3}} S_{u(\lambda)}: S_{\bar{u}(\lambda)}\right) \\
& -\beta \Lambda_{k}\left\|\Phi_{0}\right\|_{L^{2}}^{2} \operatorname{Re}\left(\partial_{\lambda} \mu(\lambda)\right)-\lambda_{2}^{1} \partial_{\lambda}|\mu(\lambda)|^{2}\left\|\Phi_{0}\right\|_{L^{2}}^{2} \\
= & \frac{1}{2} \partial_{\lambda}\left\{\operatorname{Re}(\mu(\lambda)) 2 \beta \Lambda_{k}\left\|\Phi_{0}\right\|_{L^{2}}^{2}+\lambda|\mu(\lambda)|^{2}\left\|\Phi_{0}\right\|_{L^{2}}^{2}\right\} \\
& -\beta \Lambda_{k}\left\|\Phi_{0}\right\|_{L^{2}}^{2} \operatorname{Re}\left(\partial_{\lambda} \mu(\lambda)\right)-\lambda_{2}^{1} \partial_{\lambda}|\mu(\lambda)|^{2}\left\|\Phi_{0}\right\|_{L^{2}}^{2} \\
= & \frac{1}{2}|\mu(\lambda)|^{2}\left\|\Phi_{0}\right\|_{L^{2}}^{2} .
\end{aligned}
$$

Using $\operatorname{Re}\left\langle u, \partial_{\lambda} u\right\rangle=0$, we arrive at

$$
\operatorname{Im}\left\langle u, \partial_{\lambda} u\right\rangle \operatorname{Im}(\mu)=-\frac{1}{2}|\mu(\lambda)|^{2}\left\|\Phi_{0}\right\|_{L^{2}}^{2} .
$$

Now we differentiate the eigenvalue equation with respect to $\lambda$, multiply with $\partial_{\lambda} u$, and take the imaginary part:

$$
\begin{aligned}
0 & =\operatorname{Im}\left\langle\partial_{\lambda}(-\mu u), \partial_{\lambda} u\right\rangle+\operatorname{Im}\left\langle-\nu \Delta \partial_{\lambda} u+\nabla \partial_{\lambda} p, \partial_{\lambda} u\right\rangle \\
& =-\operatorname{Re}\left(\partial_{\lambda} \mu\right) \operatorname{Im}\left\langle u, \partial_{\lambda} u\right\rangle-\operatorname{Im}(\mu)\left\|\partial_{\lambda} u\right\|^{2}-\operatorname{Im}\left\{\partial_{\lambda}\left(\beta \Lambda_{k}+\lambda \mu\right) \partial_{\lambda} \bar{\mu}\right\}\left\|\Phi_{0}\right\|_{L^{2}}^{2} .
\end{aligned}
$$

Multiplying with $2 \operatorname{Im}(\mu)$ and inserting (42) yields

$$
\operatorname{Re}\left(\partial_{\lambda} \mu\right)|\mu|^{2}\left\|\Phi_{0}\right\|_{L^{2}}^{2}-2|\operatorname{Im}(\mu)|^{2}\left\|\partial_{\lambda} u\right\|^{2}=2 \operatorname{Im}(\mu) \operatorname{Im}\left(\mu \partial_{\lambda} \bar{\mu}\right)\left\|\Phi_{0}\right\|_{L^{2}}^{2} .
$$

On the imaginary axis, $\operatorname{Re}(\mu)=0$, this formula simplifies to

$$
-\operatorname{Re}\left(\partial_{\lambda} \mu\right)\left\|\Phi_{0}\right\|_{L^{2}}^{2}=2\left\|\partial_{\lambda} u\right\|^{2} .
$$

It proves transversality and the direction of the crossing.

The above results lead to a Hopf bifurcation. Due to the restricted regularity, one has to avoid the implicit function theorem in the proof and use degree theory. It allows us to replace the assumption of transversality by the following. The eigenvalue cannot follow the imaginary axis, i.e.,

$$
\mu(\bar{\lambda}) \in i \mathbb{R} \Rightarrow \forall \varepsilon>0: \operatorname{Re}(\mu(\bar{\lambda}-\varepsilon))>0, \quad \operatorname{Re}(\mu(\bar{\lambda}+\varepsilon))<0 .
$$

THEOREM 6.4. For fixed wave number $k_{0}$, there exists a critical value for $\lambda$ such that a pair of eigenvalues $\mu^{ \pm}$of $\mathcal{L}_{\lambda}$ are purely imaginary. Assume that there is no resonance, i.e., the eigenvalues for different $k$ are no integer multiples of $\mu^{+}$. Then 
a Hopf bifurcation occurs and there exists a continuous branch of $O(2)$-symmetric, periodic solutions of the nonlinear equations.

Proof. In Proposition 4.3 we parametrized small initial values for the nonlinear equation over $U=B_{\varepsilon}(0) \subset D_{\mathcal{L}, \alpha}$ with a mapping $\zeta: U \rightarrow X^{4}$. We want to restrict ourselves to functions of the prescribed symmetry group $\Gamma \simeq O(2)$. We parametrize small compatible initial values with symmetry $\Gamma$ over $V:=U \cap$ Fix $(\Gamma)$ with a map $\zeta_{\Gamma}: V \rightarrow F i x(\Gamma) \subset X^{4}$ and consider the flow

$$
\Phi: V \times \mathbb{R} \times \mathbb{R} \ni(z, t, \lambda) \mapsto \zeta_{\Gamma}^{-1} x(t)-z \in V .
$$

Here $x(t)$ is the solution of the nonlinear equation with parameter $\lambda$ to the initial value $\zeta_{\Gamma}(z)$. We used the fact that the nonlinear equation preserves the $\Gamma$-symmetry. We want to solve $\Phi(z, t, \lambda)=z$ with nontrivial $z$. The linearization of $\Phi$ in $(0, \bar{t}=$ $\left.\underset{\operatorname{Im}\left(\mu_{0}(\bar{\lambda})\right)}{2 \pi}, \bar{\lambda}\right)$ is

$$
D_{z} \Phi: V \rightarrow V, \quad z \mapsto e^{-\mathcal{L}_{\bar{\lambda}} \bar{t}} z,
$$

and the kernel of $D_{z} \Phi(., \bar{t}, \bar{\lambda})-i d$ is two-dimensional. One can perform a LiapunovSchmidt reduction and solve the bifurcation equation with degree theory.

7. Water waves generated by wind. We analyze a simple two-dimensional model for a wind-generated instability of a water surface. The wind changes the pressure along the surface. We assume that the pressure profile follows the sinusoidal profile of the surface and is shifted by an angle $\phi$. Measurements of Elliott [7] justify this assumption and give the value of $135^{\circ}$ for $\phi$.

We mention the two major simplifications of this model: it neglects the tangential stress, and we linearize about the zero-solution instead of assuming an underlying shear flow. The method could be extended, but the desirable further development would be a two-phase model to explore the dependence of the force of the wave number. For qualitative studies we refer to [12] and references therein.

With a surface elevation $\eta$ we write the additional pressure as $e^{i \phi} \beta^{*} \eta$. This is equivalent to saying that we treat the complex surface tension $\beta+e^{i \phi} \beta^{*} \Lambda_{k}^{-1}$. We fix the direction of the wind by setting $0<\phi<\pi$. The eigenvalue equations are

$$
\begin{aligned}
\lambda u+\nu \Delta u-\nabla p & =0, \\
\nabla \cdot u & =0, \\
\left.u_{n}\right|_{\partial} & =-\lambda \eta, \\
\left.\left(p-2 \nu S_{u}^{n}\right)\right|_{\partial} & =-\beta \Delta \eta+e^{i \phi} \beta^{*} \eta .
\end{aligned}
$$

Every eigenspace is (at least) two-dimensional due to the equivariance under translations. Choosing $\eta(x)=\Phi_{0}(x)=e^{i k x}$, we select one of the eigenfunctions. On the other hand, a positive $\beta^{*}$ destroys the symmetry of reflections $x \mapsto-x$. The conjugate complex of an eigenvalue need not be an eigenvalue for the same $\eta$.

Lemma 7.1. For positive $\beta^{*}$ there is precisely one eigenvalue with positive imaginary part. The other eigenvalues are below the real axis and have a positive real part.

Proof. As in section 5 we can write the eigenvalue equation as

$$
\tilde{r}(z)=\Lambda_{k} \beta+e^{i \phi} \beta^{*} .
$$

The proof of the lemma is based on the study of $\tilde{r}$ in Proposition 5.3. 
For $\beta^{*}=0$ we consider two cases.

Case 1 . The surface tension is above its critical value. Then there are two nonreal eigenvalues with multiplicity 2. Due to the reflection symmetry, they can both be represented with an eigenfunction with $\eta(x)=\Phi_{0}(x)$.

The function $\tilde{r}: \mathbb{R} \rightarrow \mathbb{R}$ was shown to have a negative derivative in the real eigenvalues. Since the function $\tilde{r}$ is analytic in a neighborhood of the eigenvalue and $\beta^{*}$ acts like an imaginary component of the surface tension, we conclude that for small, positive $\beta^{*}$ the eigenvalues get a negative imaginary part.

Case 2. The surface tension is below its critical value. Then the derivative of $\tilde{r}: \mathbb{R} \rightarrow \mathbb{R}$ is positive in the first eigenvalue, negative in all other eigenvalues. The same reasoning as before proves that for small positive $\beta^{*}$ the first eigenvalue gets a positive imaginary part, and the other eigenvalues get a negative imaginary part.

In both cases a return to the real axis is not possible for finite $\beta^{*}$ since real eigenvalues correspond to real surface tension or $\beta^{*}=0$.

We derive an equation for $\lambda$ by testing the eigenvalue equation with $(u, \eta)$ in the energy space and using the integration by parts:

$$
\begin{aligned}
\int(-\nu \Delta u+\nabla p) \bar{u} & =2 \nu \int\left|S_{u}\right|^{2}+\int_{\partial}\left(p-2 \nu S_{u}^{n}\right) \bar{u}_{n} \\
& =2 \nu \int\left|S_{u}\right|^{2}-\int_{\partial}\left(-\beta \Delta \eta+e^{i \phi} \beta^{*} \eta\right) \bar{\lambda} \bar{\eta} .
\end{aligned}
$$

In analogy to Lemma 2.4, we get

$$
\lambda\left\{\|u\|^{2}+\|\eta\|_{E}^{2}\right\}=2 \nu \int\left|S_{u}\right|^{2}+2 i \operatorname{Im} \lambda\|\eta\|_{E}^{2}-e^{i \phi} \beta^{*} \bar{\lambda}\|\eta\|_{L^{2}}^{2} .
$$

Taking the real part of this equation, we get

$$
\operatorname{Re} \lambda=0 \Rightarrow 0<\operatorname{Re}\left(e^{-i \phi} \lambda\right) \Rightarrow \operatorname{Im}(\lambda)>0 .
$$

This proves that the eigenvalues with negative imaginary part cannot cross the imaginary axis.

The only eigenvalue that can create an instability is the one with positive imaginary part, further denoted by $\lambda^{+}$. We turn to an analysis of this eigenvalue.

We know that for eigenvalues $\lambda$ in a compact subset of $\mathbb{C}-\left\{\kappa_{j} \mid j \in \mathbb{N}\right\}$ the corresponding values of $\beta^{*}=e^{-i \phi}\left(\tilde{r}(\lambda)-\Lambda_{k} \beta\right)$ are finite. Therefore, for $\beta^{*} \rightarrow \infty$,

$$
\begin{aligned}
\exists j: \quad \lambda^{+}\left(\beta^{*}\right) & \rightarrow \kappa_{j} \\
\text { or } \quad\left|\lambda^{+}\left(\beta^{*}\right)\right| & \rightarrow \infty .
\end{aligned}
$$

We take the imaginary part of (49) and get

$$
\operatorname{Im} \lambda^{+}\left\{\|u\|^{2}-\|\eta\|_{E}^{2}\right\}=-\beta^{*} \operatorname{Im}\left(e^{i \phi} \bar{\lambda}^{+}\right)\|\eta\|_{L^{2}}^{2} .
$$

Assume that $\operatorname{Im}\left(\lambda^{+}\right)$stays bounded. Then we know $\left|\operatorname{Re}\left(\lambda^{+}\right)\right| \rightarrow \infty$. The left-hand side of (50) is bounded from below. We conclude that $\operatorname{Re}\left(\lambda^{+}\right) \rightarrow-\infty$.

The eigenvalue must cross the imaginary axis, by the following lemma.

Lemma 7.2. An eigenvalue $\lambda$ with $|\operatorname{Im} \lambda| \rightarrow \infty$ or $\operatorname{Re}(\lambda) \rightarrow-\infty$ as $\beta^{*} \rightarrow \infty$ satisfies

$$
\arg (\lambda) \rightarrow \frac{\phi \pm \pi}{2} \quad \text { for } \beta^{*} \rightarrow \infty
$$


Proof. To prove this proposition we use the explicit formulas for eigenfunctions. We introduce

$$
v=u-\frac{1}{\lambda} \nabla p,
$$

which solves $\lambda v+\nu \Delta v=0$. With constants $A=\left(A_{1}, A_{2}\right), B=\left(B_{1}, B_{2}\right), P$, and $Q$ we write

$$
\begin{aligned}
p(x, y) & =P e^{k y} e^{i k x}+Q e^{-k y} e^{i k x}, \\
v(x, y) & =A e^{\mu y} e^{i k x}+B e^{-\mu y} e^{i k x}, \\
\mu^{2} & =k^{2}-\frac{\lambda}{\nu},
\end{aligned}
$$

where we take $\mu$ as the root with positive real part, $\operatorname{Re} \mu \rightarrow \infty$.

The incompressibility reads

$$
i k A_{1}+\mu A_{2}=0, \quad i k B_{1}-\mu B_{2}=0 .
$$

We get an equation for $P$ and $Q$ if we construct

$$
\begin{aligned}
0 & =i k u_{1}(-h)+\mu u_{2}(-h) \\
& =\frac{k^{2}}{\lambda}\left(P e^{-k h}+Q e^{k h}\right)-\frac{k \mu}{\lambda}\left(P e^{-k h}-Q e^{k h}\right),
\end{aligned}
$$

which proves that $|Q / P|$ is bounded, and then

$$
\left(1-\frac{Q}{P} e^{2 k h}\right)=\frac{1}{P}\left(P-Q e^{2 k h}\right) \rightarrow 0 .
$$

Using $u(x,-h)=0$, this implies

$$
\frac{B_{1}}{\left|A_{1}\right|+|P|} \rightarrow 0 \quad \text { and } \quad \frac{B_{2}}{\left|A_{2}\right|+|P|} \rightarrow 0 \quad \text { exponentially in } \mu .
$$

We use the boundary condition of vanishing tangential stress,

$$
0=A_{1} \mu-B_{1} \mu+A_{2} i k+B_{2} i k+\frac{2 i k^{2}}{\lambda}(P-Q),
$$

to conclude that

$$
\frac{A_{1} \mu \lambda}{i k^{2}(P-Q)} \rightarrow-1
$$

The boundary condition for the normal stress reads

$$
0=(P+Q)-2 \nu\left(A_{2}-B_{2}\right) \mu-\frac{2 \nu}{\lambda} k^{2}(P+Q)-\beta \Lambda_{k}-e^{i \phi} \beta^{*} .
$$

Collecting the dominant terms yields

$$
\frac{P+Q}{\beta^{*}} \rightarrow e^{i \phi} .
$$


The kinematic boundary equation is

$$
A_{2}+B_{2}+\frac{k}{\lambda}(P-Q)=-\lambda .
$$

Using (54), this implies

$$
\frac{k}{\lambda^{2}}(P-Q) \rightarrow-1
$$

Combining (53), (55), and (56) gives

$$
\frac{\lambda^{2}}{e^{i \phi} \beta^{*}} \rightarrow-k \frac{1-e^{-2 k h}}{1+e^{-2 k h}} .
$$

REMARK 7.3. The basic idea in the above proof was to show that the dominating term in the stress equation is the pressure. In this sense the following formal calculation for infinite height and vanishing viscosity is justified:

$$
\begin{gathered}
\Delta p=0,\left.\quad p\right|_{\partial}=e^{i \phi} \beta^{*} \eta, \quad u=\frac{1}{\lambda} \nabla p, \\
-\lambda \eta=\left.u_{n}\right|_{\partial}=\frac{1}{\lambda} \partial_{n} p=\frac{k}{\lambda} e^{i \phi} \beta^{*} \eta .
\end{gathered}
$$

REMARK 7.4. To reduce the formula to the case of vanishing wind but variable surface tension $T$, one can insert $\beta^{*}=\Lambda_{k} T=k^{2} T$ to get the asymptotic formula

$$
(-i \lambda)^{2}=T k^{3} \frac{1-e^{-2 k h}}{1+e^{-2 k h}}
$$

Formulas that include the viscous effect and give a similar expression for the real part of $\lambda$ can be found in [11].

In this example it is not easy to prove transversality of the crossing. On the other hand, we can easily verify property (43). If the eigenvalue followed the imaginary axis then $e^{-i \phi} \tilde{r}(z)$ were real on an interval of the imaginary axis. By analyticity, it would be real on the whole of the imaginary axis, which contradicts the last lemma.

With the proof of the last section we arrived at the following theorem.

THEOREM 7.5. Assuming the nonresonance condition, for some critical value of $\beta^{*}$ a Hopf bifurcation occurs and periodic solutions of the nonlinear evolution equations exist.

We remark that the constructed solution is a propagating wave but not necessarily a travelling wave.

\section{REFERENCES}

[1] S. Agmon, A. Douglis, And N. Nirenberg, Estimates near the boundary for solutions of elliptic partial differential equations satisfying general boundary conditions II, Comm. Pure Appl. Math., 17 (1964), pp. 35-92.

[2] J. T. BeAle, Large time regularity of viscous surface waves, Arch. Rational Mech. Anal., 84 (1984), pp. 307-352.

[3] E. Becker, W. J. Hiller, And T. A. Kowalewski, Nonlinear dynamics of viscous droplets, J. Fluid Mech., 258 (1994), pp. 191-216. 
[4] J. Bemelmans, Gleichgewichtsfiguren zäher Flüssigkeiten mit Oberflächenspannung, Analysis, 1 (1981), pp. 241-282.

[5] M. G. Crandall and P. H. Rabinowitz, The Hopf bifurcation theorem in infinite dimensions, Arch. Rational Mech. Anal., 67 (1978), pp. 53-72.

[6] G. DA PRATo, Abstract differential equations, maximal regularity, and linearization, Nonlinear Functional Analysis and Its Applications, Proc. Symp. in Pure Math., 45/1 (1986), pp. 359370.

[7] J. A. ElLiott, Microscale pressure fluctuations near waves generated by wind, J. Fluid Mech., 54 (1972), pp. 427-448.

[8] M. Golubitsky, I. Stewart, And D. Schaeffer, Singularities and Groups in Bifurcation Theory 2, Applied Mathematical Sciences 69, Springer-Verlag, Berlin, 1988.

[9] H. Koch, On a Fully Nonlinear Mixed Parabolic Problem with Oblique Boundary Condition, Preprintserie des IWR 95-20, Heidelberg, Germany, 1995.

[10] H. LAMB, Lehrbuch der Hydrodynamik, Lehrbuch der mathematischen Naturwissenschaften, 26, Teubner, Leipzig, 1931.

[11] T. S. Lundgren And N. N. Mansour, Oscillations of drops in zero gravity with weak viscous effects, J. Fluid Mech., 194 (1988), pp. 479-510.

[12] L. C. Morland and P. N. Saffman, Effect of wind profile on the instability of wind blowing over water, J. Fluid Mech., 252 (1993), pp. 383-398.

[13] M. Renardy and D. D. Joseph, Hopf bifurcation in two component flow, SIAM J. Math. Anal., 17 (1986), pp. 894-910.

[14] B. Schweizer, Oscillatory behavior of liquid drops, Preprintserie des IWR 95-34, Heidelberg, Germany, 1995.

[15] V. A. Solonnikov, The solvability of the problem concerning the evolution of an isolated volume of viscous incompressible capillary fluid, J. Soviet Math., 32 (1986), pp. 223-228.

[16] V. A. Solonnikov And V. Skadilov, On a boundary value problem for a stationary system of the Navier-Stokes equations, Proc. Steklov Inst. Math., 125 (1973), pp. 186-199.

[17] A. Wagner, Stationary Marangoni Convection: A Free Boundary Problem for the Navier Stokes Equations, Preprintserie des IWR 94-41, Heidelberg, Germany, 1994. 